\title{
Asymptotic Behavior of Global Solutions to the Boussinesq Equation in Multidimensions
}

\author{
Yu-Zhu Wang and Qingnian Zhang \\ School of Mathematics and Information Sciences, North China University of Water Resources and Electric Power, \\ Zhengzhou 450011, China
}

Correspondence should be addressed to Yu-Zhu Wang; yuzhu108@163.com and Qingnian Zhang; qingnianzhang62@163.com Received 30 May 2013; Revised 16 September 2013; Accepted 20 September 2013

Academic Editor: Shaoyong Lai

Copyright (C) 2013 Y.-Z. Wang and Q. Zhang. This is an open access article distributed under the Creative Commons Attribution License, which permits unrestricted use, distribution, and reproduction in any medium, provided the original work is properly cited.

The Cauchy problem for the Boussinesq equation in multidimensions is investigated. We prove the asymptotic behavior of the global solutions provided that the initial data are suitably small. Moreover, our global solutions can be approximated by the solutions to the corresponding linear equation as time tends to infinity when the dimension of space $n \geq 3$.

\section{Introduction}

We investigate the Cauchy problem of the following damped Boussinesq equation in multidimensions:

$$
u_{t t}-a \Delta u_{t t}-2 b \Delta u_{t}-\alpha \Delta^{3} u+\beta \Delta^{2} u-\Delta u=\Delta f(u)
$$

with the initial value

$$
t=0: u=u_{0}(x), \quad u_{t}=u_{1}(x) .
$$

Here $u=u(x, t)$ is the unknown function of $x=$ $\left(x_{1}, \ldots, x_{n}\right) \in \mathbb{R}^{n}$ and $t>0, a, b, \alpha$, and $\beta$ are positive constants. The nonlinear term $f(u)=O\left(u^{2}\right)$.

When $f(u)=u^{2}$, (1) has been studied by several authors. The authors investigated the first initial boundary value problem for (1) in a unit circle (see [1]). The existence and the uniqueness of strong solution were established and the solutions were constructed in the form of series in the small parameter present in the initial conditions. The longtime asymptotic was also obtained in the explicit form. The authors considered the initial-boundary value problem for (1) in the unit ball $B \subset \mathbb{R}^{3}$, similar results were established in [2].

Recently, Wang [3] proved the global existence and asymptotic decay of solutions to the problem (1), (2). Their proof is based on the contraction mapping principle and makes use of the sharp decay estimates for the linearized problem. The main purpose of this paper is to establish the following optimal decay estimate of solutions to (1) and (2) by constructing the antiderivatives conditions:

$$
u_{1}(x)=\partial_{x_{1}} v_{1}(x) \text {. }
$$

Then we obtain a better decay rate of solutions than the previous one in [3]. Moreover, our global solutions can be approximated by the solutions to the corresponding linear equation. The decay estimate is said to be optimal because we have used the sharp decay estimates for the solution operators $G(x, t)$ and $H(x, t)$, which are defined by (15) and (16), respectively. Since the solution operator $G(x, t)$ has singularity, therefore, we construct the antiderivatives conditions $u_{1}(x)=\partial_{x_{1}} v_{1}(x)$ and eliminate the singularity and obtain the same decay estimate for the solution operators $H(x, t)$. For details; see Lemma 4. The study of the global existence and asymptotic behavior of solutions to wave equations has a long history. We refer to [4-10] for wave equations. Now we state our results as follows.

Theorem 1. Let $s \geq[n / 2]-1$ and let $n \geq 2$. Assume that $u_{0} \in H^{s+2}\left(\mathbb{R}^{n}\right), v_{1} \in H^{s+1}\left(\mathbb{R}^{n}\right)$. Put

$$
E_{0}=\left\|u_{0}\right\|_{H^{s+2}}+\left\|v_{1}\right\|_{H^{s+1}} \text {. }
$$

If $E_{0}$ is suitably small, the Cauchy problem (1), (2) has a unique global solution $u(x, t)$ satisfying

$$
u \in C\left([0, \infty) ; H^{s+2}\right) \bigcap C^{1}\left([0, \infty) ; H^{s}\right) .
$$


Moreover, the solution satisfies the decay estimate:

$$
\begin{gathered}
\left\|\partial_{x}^{k} u(t)\right\|_{L^{2}} \leq C E_{0}(1+t)^{-(k / 2)}, \\
\left\|\partial_{x}^{k} u_{t}(t)\right\|_{L^{2}} \leq C E_{0}(1+t)^{-((k+1) / 2)}
\end{gathered}
$$

for $0 \leq k \leq s+2$ in (6) and $0 \leq k \leq s$ in (7).

From the proof of Theorem 1, we have the following corollary immediately.

Corollary 2. Let $n \geq 3$ and assume the same conditions of Theorem 1. Then the solution $u$ of the problem (1), (2), which is constructed in Theorem 1, can be approximated by the linear solution $u_{L}$ as $t \rightarrow \infty$. In fact, we have

$$
\begin{gathered}
\left\|\partial_{x}^{k}\left(u-u_{L}\right)(t)\right\|_{L^{2}} \leq C E_{0}^{2}(1+t)^{-(k / 2)} \eta(t), \\
\left\|\partial_{x}^{k}\left(u-u_{L}\right)_{t}(t)\right\|_{L^{2}} \leq C E_{0}^{2}(1+t)^{-((k+1) / 2)} \eta(t)
\end{gathered}
$$

for $0 \leq k \leq s+1$ and $0 \leq k \leq s$, respectively, where $u_{L}(t):=G(t) * \partial_{x_{1}} v_{1}+H(t) * u_{0}$ is the linear solution and $\eta(t)=(1+t)^{-((n-2) / 4)}$. Here $G(t)$ and $H(t)$ are given by $(15)$ and (16), respectively.

Notations. For $1 \leq p \leq \infty, L^{p}=L^{p}\left(\mathbb{R}^{n}\right)$ denotes the usual Lebesgue space with the norm $\|\cdot\|_{L^{p}}$. The usual Sobolev space of order $s$ is defined by $W^{s, p}=(I-\Delta)^{-(s / 2)} L^{p}$ with the norm $\|f\|_{W^{s, p}}=\left\|(I-\Delta)^{s / 2} f\right\|_{L^{p}}$. The corresponding homogeneous Sobolev space of order $s$ is defined by $\dot{W}^{s, p}=(-\Delta)^{-(s / 2)} L^{p}$ with the norm $\|f\|_{\dot{W}^{s, p}}=\left\|(-\Delta)^{s / 2} f\right\|_{L^{p}}$; when $p=2$, we write $H^{s}=W^{s, 2}$ and $\dot{H}^{s}=\dot{W}^{s, 2}$. We note that $W^{s, p}=L^{p} \cap \dot{W}^{s, p}$ for $s \geq 0$.

The plan of the paper is arranged as follows. In Section 2 we derive the solution formula of the problem (1), (2) and prove the decay property of the solution operators appearing in the solution formula. Then, in Sections 3, we prove the optimal asymptotic decay of solutions to the problem (1), (2).

\section{Decay Property}

The aim of this section is to derive the solution formula for the problem (1), (2). We first investigate the linear equation of (1):

$$
\begin{gathered}
u_{t t}-a \Delta u_{t t}-2 b \Delta u_{t}-\alpha \Delta^{3} u \\
+\beta \Delta^{2} u-\Delta u=0 .
\end{gathered}
$$

With the initial data (2). Taking the Fourier transform, we have

$$
\begin{array}{r}
\quad\left(1+a|\xi|^{2}\right) \widehat{u}_{t t}+2 b|\xi|^{2} \widehat{u}_{t} \\
+\left(\alpha|\xi|^{6}+\beta|\xi|^{4}+|\xi|^{2}\right) \widehat{u}=0 ; \\
t=0: \widehat{u}=\widehat{u}_{0}(\xi), \quad \widehat{u}_{t}=i \xi_{1} \widehat{v}_{1}(\xi) .
\end{array}
$$

The characteristic equation of (10) is

$$
\left(1+a|\xi|^{2}\right) \lambda^{2}+2 b|\xi|^{2} \lambda+\alpha|\xi|^{6}+\beta|\xi|^{4}+|\xi|^{2}=0
$$

Let $\lambda=\lambda_{ \pm}(\xi)$ be the corresponding eigenvalues of (12), we obtain

$\lambda_{ \pm}(\xi)$

$$
=\frac{-b|\xi|^{2} \pm|\xi| \sqrt{-1-\left(a+\beta-b^{2}\right)|\xi|^{2}-(\alpha+a \beta)|\xi|^{4}-a \alpha|\xi|^{6}}}{1+a|\xi|^{2}} .
$$

The solution to the problem (10), (11) is given in the form

$$
\widehat{u}(\xi, t)=\widehat{G}(\xi, t) i \xi_{1} \widehat{v}_{1}(\xi)+\widehat{H}(\xi, t) \widehat{u}_{0}(\xi),
$$

where

$$
\begin{gathered}
\widehat{G}(\xi, t)=\frac{1}{\lambda_{+}(\xi)-\lambda_{-}(\xi)}\left(e^{\lambda_{+}(\xi) t}-e^{\lambda_{-}(\xi) t}\right) \\
\widehat{H}(\xi, t)=\frac{1}{\lambda_{+}(\xi)-\lambda_{-}(\xi)}\left(\lambda_{+}(\xi) e^{\lambda_{-}(\xi) t}-\lambda_{-}(\xi) e^{\lambda_{+}(\xi) t}\right) .
\end{gathered}
$$

We define $G(x, t)$ and $H(x, t)$ by $G(x, t)=F^{-1}[\widehat{G}(\xi, t)](x)$ and $H(x, t)=F^{-1}[\widehat{H}(\xi, t)](x)$, respectively, where $F^{-1}$ denotes the inverse Fourier transform. Then, applying $F^{-1}$ to (14), we obtain

$$
u(t)=G(t) * \partial_{x_{1}} v_{1}+H(t) * u_{0} .
$$

By the Duhamel principle, we obtain the solution formula to (1), (2) as

$$
\begin{aligned}
u(t)= & G(t) * \partial_{x_{1}} v_{1}+H(t) * u_{0} \\
& +\int_{0}^{t} G(t-\tau) *(I-a \Delta)^{-1} \Delta f(u)(\tau) d \tau .
\end{aligned}
$$

In what follows, the aim is to establish decay estimates of the solution operators $G(t)$ and $H(t)$ appearing in the solution formula (18). Firstly, we state the pointwise estimate of solutions in the Fourier space. The result can be found in $[3]$.

Lemma 3. The solution of the problem (10), (11) satisfies

$$
\begin{aligned}
&|\xi|^{2}\left(1+|\xi|^{2}\right)|\widehat{u}(\xi, t)|^{2}+\left|\widehat{u}_{t}(\xi, t)\right|^{2} \\
& \leq C e^{-c \omega(\xi) t}\left(|\xi|^{2}\left(1+|\xi|^{2}\right)\left|\widehat{u}_{0}(\xi)\right|^{2}\right. \\
&\left.+\left|\xi_{1}\right|^{2}\left|\widehat{v}_{1}(\xi)\right|^{2}\right),
\end{aligned}
$$

for $\xi \in \mathbb{R}^{n}$ and $t \geq 0$, where $\omega(\xi)=|\xi|^{2} /\left(1+|\xi|^{2}\right)$.

From Lemma 3, we immediately get the following. 
Lemma 4. Let $\widehat{G}(\xi, t)$ and $\widehat{H}(\xi, t)$ be the fundamental solution of (10) in the Fourier space, which are given in (15) and (16), respectively. Then we have the estimates

$$
\begin{aligned}
&|\xi|^{2}(1\left.+|\xi|^{2}\right)|\widehat{G}(\xi, t)|^{2}+\left|\widehat{G}_{t}(\xi, t)\right|^{2} \\
& \leq C e^{-c \omega(\xi) t} \\
&|\xi|^{2}(1\left.+|\xi|^{2}\right)|\widehat{H}(\xi, t)|^{2}+\left|\widehat{H}_{t}(\xi, t)\right|^{2} \\
& \quad \leq C|\xi|^{2}\left(1+|\xi|^{2}\right) e^{-c \omega(\xi) t}
\end{aligned}
$$

for $\xi \in \mathbb{R}^{n}$ and $t \geq 0$, where $\omega(\xi)=|\xi|^{2} /\left(1+|\xi|^{2}\right)$.

Lemma 5. Let $k, j, l$ be nonnegative integers and let $1 \leq p \leq$ 2. Then we have

$$
\begin{aligned}
\| \partial_{x}^{k} G(t) & * \partial_{x_{1}} \phi \|_{L^{2}} \\
\leq & C(1+t)^{-(n / 2)((1 / p)-(1 / 2))-((k-j) / 2)}\left\|\partial_{x}^{j} \phi\right\|_{L^{p}} \\
& +C e^{-c t}\left\|\partial_{x}^{k+l-1} \phi\right\|_{L^{2}} \\
\| \partial_{x}^{k} H(t) & * \psi \|_{L^{2}} \\
\leq & C(1+t)^{-(n / 2)((1 / p)-(1 / 2))-((k-j) / 2)}\left\|\partial_{x}^{j} \psi\right\|_{L^{p}} \\
& +C e^{-c t}\left\|\partial_{x}^{k+l} \psi\right\|_{L^{2}}
\end{aligned}
$$

for $0 \leq j \leq k$, where $k+l-1 \geq 0$ in (22). Similarly, we have

$$
\begin{aligned}
\| \partial_{x}^{k} G_{t}(t) & * \partial_{x_{1}} \phi \|_{L^{2}} \\
\leq & C(1+t)^{-(n / 2)((1 / p)-(1 / 2))-((k+1-j) / 2)}\left\|\partial_{x}^{j} \phi\right\|_{L^{p}} \\
& +C e^{-c t}\left\|\partial_{x}^{k+l+1} \phi\right\|_{L^{2}}, \\
\left\|\partial_{x}^{k} H_{t}(t) * \psi\right\|_{L^{2}} & \\
\leq & C(1+t)^{-(n / 2)((1 / p)-(1 / 2))-((k+1-j) / 2)}\left\|\partial_{x}^{j} \psi\right\|_{L^{p}} \\
& +C e^{-c t}\left\|\partial_{x}^{k+l+2} \psi\right\|_{L^{2}}
\end{aligned}
$$

for $0 \leq j \leq k+1$.

Proof. We only prove (22). By the Plancherel theorem and (20), we obtain

$$
\begin{aligned}
\| \partial_{x}^{k} G(t) & * \partial_{x_{1}} \phi \|_{L^{2}}^{2} \\
= & \int_{|\xi| \leq 1}|\xi|^{2 k}|\widehat{G}(\xi, t)|^{2}\left|\xi_{1}\right|^{2}|\widehat{\phi}(\xi)|^{2} d \xi \\
& \quad+\int_{|\xi| \geq 1}|\xi|^{2 k}|\widehat{G}(\xi, t)|^{2}\left|\xi_{1}\right|^{2}|\widehat{\phi}(\xi)|^{2} d \xi
\end{aligned}
$$

$$
\begin{aligned}
\leq & C \int_{|\xi| \leq 1}|\xi|^{2 k} e^{-c|\xi|^{2} t}|\widehat{\phi}(\xi)|^{2} d \xi \\
& +C e^{-c t} \int_{|\xi| \geq 1}|\xi|^{2 k+2}\left(|\xi|^{2}\left(1+|\xi|^{2}\right)\right)^{-1}|\widehat{\phi}(\xi)|^{2} d \xi \\
= & : I_{1}+I_{2} .
\end{aligned}
$$

For the term $I_{1}$, letting $1 / p^{\prime}+1 / p=1$, we have

$$
\begin{aligned}
I_{1} & \leq C \int_{|\xi| \leq 1}|\xi|^{2 k} e^{-c|\xi|^{2} t}|\widehat{\phi}(\xi)|^{2} d \xi \\
& \leq C\left\||\xi|^{j} \hat{\phi}\right\|_{L^{p^{\prime}}}^{2}\left(\int_{|\xi| \leq 1}|\xi|^{2(k-j) p} e^{-c q|\xi|^{2} t} d \xi\right)^{1 / p} \\
& \leq C(1+t)^{-n((1 / p)-(1 / 2))-(k-j)}\left\|\partial_{x}^{j} \phi\right\|_{L^{p}}^{2}
\end{aligned}
$$

where we used the Hölder inequality with $\left(2 / p^{\prime}\right)+(1 / q)=1$ and the Hausdorff-Young inequality $\|\widehat{v}\|_{L^{p^{\prime}}} \leq C\|v\|_{L^{p}}$ for $v=$ $(-\Delta)^{-(1 / 2)} \partial_{x}^{j} \phi$. On the other hand, we can estimate the term $I_{2}$ simply as

$$
\begin{aligned}
I_{2} & \leq C e^{-c t} \int_{|\xi| \geq 1}|\xi|^{2 k-2}|\widehat{\phi}(\xi)|^{2} d \xi \\
& \leq C e^{-c t} \int_{|\xi| \geq 1}|\xi|^{2(k+l-1)}|\widehat{\phi}(\xi)|^{2} d \xi \\
& \leq C e^{-c t}\left\|\partial_{x}^{k+l-1} \phi\right\|_{L^{2}}^{2},
\end{aligned}
$$

where $k+l-1 \geq 0$. Combining (26)-(28) yields (22). We have completed the proof of the Lemma.

Similar to the proof of Lemma 5, it is not difficult to get the following.

Lemma 6. Let $1 \leq p \leq 2$ and let $k, j, l$ be nonnegative integers. Then we have the following estimate:

$$
\begin{aligned}
\| \partial_{x}^{k} G(t) & *(I-a \Delta)^{-1} \Delta g \|_{L^{2}} \\
\leq & C(1+t)^{-(n / 2)((1 / p)-(1 / 2))-((k+1-j) / 2)}\left\|\partial_{x}^{j} g\right\|_{L^{p}} \\
& +C e^{-c t}\left\|\partial_{x}^{k+l} g\right\|_{L^{2}}
\end{aligned}
$$

for $0 \leq j \leq k+1$. Similarly, we have

$$
\begin{aligned}
\| \partial_{x}^{k} G_{t}(t) & *(I-a \Delta)^{-1} \Delta g \|_{L^{2}} \\
\leq & C(1+t)^{-(n / 2)((1 / p)-(1 / 2))-((k+2-j) / 2)}\left\|\partial_{x}^{j} g\right\|_{L^{p}} \\
& +C e^{-c t}\left\|\partial_{x}^{k+l} g\right\|_{L^{2}}
\end{aligned}
$$

for $0 \leq j \leq k+2$.

\section{Proof of Main Result}

In order to prove optimal decay estimate of solutions to the Cauchy problem (1), (2). We need the following Lemma, which comes from [11] (see also [12]). 
Lemma 7. Assume that $f=f(v)$ is a smooth function. Suppose that $f(v)=O\left(|v|^{1+\theta}\right)(\theta \geq 1$ is an integer $)$ when $|v| \leq v_{0}$. Then for integer $m \geq 0$, if $v \in W^{m, q}\left(\mathbb{R}^{n}\right) \bigcap L^{p}\left(\mathbb{R}^{n}\right) \bigcap L^{\infty}\left(\mathbb{R}^{n}\right)$ and $\|v\|_{L^{\infty}} \leq v_{0}$, then the following inequalities hold:

$$
\left\|\partial_{x}^{m} f(v)\right\|_{L^{r}} \leq C\|v\|_{L^{p}}\left\|\partial_{x}^{m} v\right\|_{L^{1}}\|v\|_{L^{\infty}}^{\theta-1},
$$

where $1 / r=(1 / p)+(1 / q), 1 \leq p, q, r \leq+\infty$.

Proof of Theorem 1. We can prove the existence and uniqueness of small solutions by the contraction mapping principle. Here we only show the decay estimates (6) and (7) for the solution $u$ of (18) satisfying $\|u(t)\|_{L^{\infty}} \leq M_{0}$ with some $M_{0}$. Firstly, we introduce the quantity:

$$
\mathscr{W}(t)=\sum_{k=0}^{s+2} \sup _{0 \leq \tau \leq t}(1+\tau)^{k / 2}\left\|\partial_{x}^{k} u(\tau)\right\|_{L^{2}}
$$

We apply the Gagliardo-Nirenberg inequality. This yields

$$
\|u\|_{L^{\infty}} \leq C\left\|\partial_{x}^{s_{0}} u\right\|_{L^{2}}^{\theta}\|u\|_{L^{2}}^{1-\theta},
$$

where $s_{0}=[n / 2]+1$ and $\theta=n / 2 s_{0}$. It follows from the definition of $\mathscr{W}(t)$ in (32) that

$$
\|u(t)\|_{L^{\infty}} \leq C \mathscr{W}(t)(1+t)^{-(n / 4)}
$$

provided that $s \geq[n / 2]-1$. Differentiating (18) $k$ times with respect to $x$ and taking the $L^{2}$ norm, we obtain

$$
\begin{aligned}
\left\|\partial_{x}^{k} u(t)\right\|_{L^{2}} \leq & \left\|\partial_{x}^{k} G(t) * \partial_{x_{1}} v_{1}\right\|_{L^{2}}+\left\|\partial_{x}^{k} H(t) * u_{0}\right\|_{L^{2}} \\
& +\int_{0}^{t}\left\|\partial_{x}^{k} G(t-\tau) *(I-a \Delta)^{-1} \Delta f(u(\tau))\right\|_{L^{2}} d \tau \\
= & I_{1}+I_{2}+J .
\end{aligned}
$$

Firstly, we estimate $I_{1}$. We get from (22), with $p=2, j=0$, and $l=0(l=1$ for $k=0)$,

$$
\begin{aligned}
I_{1} & \leq C(1+t)^{-(k / 2)}\left\|v_{1}\right\|_{L^{2}}+C e^{-c t}\left\|\partial_{x}^{(k-1)_{+}} v_{1}\right\|_{L^{2}} \\
& \leq C E_{0}(1+t)^{-(k / 2)},
\end{aligned}
$$

where $(k-1)_{+}=\max \{k-1,0\}$. By using (23) with $p=2$, $j=0$, and $l=0$ to the term $I_{2}$, we obtain

$$
\begin{aligned}
I_{2} & \leq C(1+t)^{-(k / 2)}\left\|u_{0}\right\|_{L^{2}}+C e^{-c t}\left\|\partial_{x}^{k} u_{0}\right\|_{L^{2}} \\
& \leq C E_{0}(1+t)^{-(k / 2)} .
\end{aligned}
$$

Next, we estimate $J$. We divide $J$ into two parts and write $J=$ $J_{1}+J_{2}$, where $J_{1}$ and $J_{2}$ are corresponding to the time intervals $[0, t / 2]$ and $[t / 2, t]$, respectively. For $J_{1}$, making use of (29) with $p=2, j=0$, and $l=0$, we arrive at

$$
\begin{aligned}
J_{1} \leq & C \int_{0}^{t / 2}(1+t-\tau)^{-((k+1) / 2)}\|f(u)(\tau)\|_{L^{2}} d \tau \\
& +C \int_{0}^{t / 2} e^{-c(t-\tau)}\left\|\partial_{x}^{k} f(u)(\tau)\right\|_{L^{2}} d \tau .
\end{aligned}
$$

By Lemma 7, we have the estimates $\|f(u)\|_{L^{2}} \leq C\|u\|_{L^{\infty}}\|u\|_{L^{2}}$ and $\left\|\partial_{x}^{k} f(u)\right\|_{L^{2}} \leq C\|u\|_{L^{\infty}}\left\|\partial_{x}^{k} u\right\|_{L^{2}}$. Thus by (34), we have

$$
\begin{gathered}
\|f(u)(\tau)\|_{L^{2}} \leq C \mathscr{W}(t)^{2}(1+\tau)^{-(n / 4)}, \\
\left\|\partial_{x}^{k} f(u)(\tau)\right\|_{L^{2}} \leq C \mathscr{W}(t)^{2}(1+\tau)^{-((n / 4)-(k / 2))} .
\end{gathered}
$$

Inserting (39) and (40) into (38) yields

$$
\begin{aligned}
J_{1} \leq & C \mathscr{W}(t)^{2} \int_{0}^{t / 2}(1+t-\tau)^{-((k+1) / 2)}(1+\tau)^{-(n / 4)} d \tau \\
& +C \mathscr{W}(t)^{2} \int_{0}^{t / 2} e^{-c(t-\tau)}(1+\tau)^{-((n / 4)-(k / 2))} d \tau \\
\leq & C \mathscr{W}(t)^{2}(1+t)^{-(k / 2)} \eta(t),
\end{aligned}
$$

where $\eta(t)=(1+t)^{-((n-2) / 4)}$. Here we assumed $n \geq 2$. For $J_{2}$, exploiting (29) with $p=2, j=k$, and $l=0$ and using (40), we deduce that

$$
\begin{aligned}
J_{2} \leq & C \int_{t / 2}^{t}(1+t-\tau)^{-(1 / 2)}\left\|\partial_{x}^{k} f(u)(\tau)\right\|_{L^{2}} d \tau \\
& +C \int_{t / 2}^{t} e^{-c(t-\tau)}\left\|\partial_{x}^{k} f(u)(\tau)\right\|_{L^{2}} d \tau \\
\leq & C \mathscr{W}(t)^{2} \int_{t / 2}^{t}(1+t-\tau)^{-(1 / 2)}(1+\tau)^{-((n / 4)-(k / 2))} d \tau \\
\leq & C \mathscr{W}(t)^{2}(1+t)^{-(k / 2)} \eta(t) .
\end{aligned}
$$

Equations (41) and (42) give

$$
J \leq C \mathscr{W}(t)^{2}(1+t)^{-((n / 4)-(k / 2))} \eta(t) .
$$

Inserting (36), (37), and (43) into (35), we obtain

$$
(1+t)^{k / 2}\left\|\partial_{x}^{k} u(t)\right\|_{L^{2}} \leq C E_{0}+C \mathscr{W}(t)^{2}
$$

for $0 \leq k \leq s+2$. Consequently, we have $\mathscr{W}(t) \leq C E_{0}+$ $C \mathscr{W}(t)^{2}$, from which we can deduce $\mathscr{W}(t) \leq C E_{0}$, provided that $E_{0}$ is suitably small. This proves the decay estimate (6).

In what follows, we prove (7). Differentiating (18) with respect to $t$ and then differentiating the resulting equation $k$ times with respect to $x$, we have

$$
\begin{aligned}
\partial_{x}^{k} u_{t}(t)= & \partial_{x}^{k} G_{t}(t) * \partial_{x_{1}} v_{1}+\partial_{x}^{k} H_{t}(t) * u_{0} \\
& +\int_{0}^{t} \partial_{x}^{k} G_{t}(t-\tau) *(I-a \Delta)^{-1} \Delta f(u)(\tau) d \tau .
\end{aligned}
$$

From (45) and Minkowski inequality, we obtain

$$
\begin{aligned}
\left\|\partial_{x}^{k} u_{t}(t)\right\|_{L^{2}} \leq & \left\|\partial_{x}^{k} G_{t}(t) * \partial_{x_{1}} v_{1}\right\|_{L^{2}}+\left\|\partial_{x}^{k} H_{t}(t) * u_{0}\right\|_{L^{2}} \\
& +\int_{0}^{t}\left\|\partial_{x}^{k} G_{t}(t-\tau) *(I-a \Delta)^{-1} \Delta f(u(\tau))\right\|_{L^{2}} d \tau \\
= & K_{1}+K_{2}+L .
\end{aligned}
$$


It follows from (24) that

$$
\begin{aligned}
K_{1} & \leq C(1+t)^{-((k+1) / 2)}\left\|v_{1}\right\|_{L^{2}}+C e^{-c t}\left\|\partial_{x}^{k+1} v_{1}\right\|_{L^{2}} \\
& \leq C E_{0}(1+t)^{-((k+1) / 2)} .
\end{aligned}
$$

By using (25), we get

$$
\begin{aligned}
K_{2} & \leq C(1+t)^{-((k+1) / 2)}\left\|u_{0}\right\|_{L^{2}}+C e^{-c t}\left\|\partial_{x}^{k+2} u_{0}\right\|_{L^{2}} \\
& \leq C E_{0}(1+t)^{-((k+1) / 2)} .
\end{aligned}
$$

Finally, we estimate $L$. Dividing $L$ into two parts and writing $L=L_{1}+L_{2}$, where $L_{1}$ and $L_{2}$ are corresponding to the time intervals $[0, t / 2]$ and $[t / 2, t]$, respectively. Firstly, we estimate the term $L_{1}$, applying (30) with $p=2, j=0$, and $l=0$ and (39), (40), we arrive at

$$
\begin{aligned}
L_{1} \leq & C \int_{0}^{t / 2}(1+t-\tau)^{-((k+2) / 2)}\|f(u)(\tau)\|_{L^{2}} d \tau \\
& +C \int_{0}^{t / 2} e^{-c(t-\tau)}\left\|\partial_{x}^{k} f(u)(\tau)\right\|_{L^{2}} d \tau \\
\leq & C \mathscr{W}^{2}(t) \int_{0}^{t / 2}(1+t-\tau)^{-((k+2) / 2)}(1+\tau)^{-(n / 4)} d \tau \\
& +C \mathscr{W}^{2}(t) \int_{0}^{t / 2} e^{-c(t-\tau)}(1+\tau)^{-((n / 4)-(k / 2))} d \tau \\
\leq & C \mathscr{W}^{2}(t)(1+t)^{-((k+1) / 2)} \eta(t) .
\end{aligned}
$$

Next, for the term $L_{1}$, it follows from (30) with $p=2, k=0$, and $l=0$ and (40) that

$$
\begin{aligned}
L_{2} \leq & C \int_{t / 2}^{t}(1+t-\tau)^{-1}\left\|\partial_{x}^{k} f(u)(\tau)\right\|_{L^{2}} d \tau \\
& +C \int_{t / 2}^{t} e^{-c(t-\tau)}\left\|\partial_{x}^{k} f(u)(\tau)\right\|_{L^{2}} d \tau \\
\leq & C \mathscr{W}^{2}(t) \int_{t / 2}^{t}(1+t-\tau)^{-1}(1+\tau)^{-((n / 4)-(k / 2))} d \tau \\
& +C \mathscr{W}^{2}(t) \int_{t / 2}^{t} e^{-c(t-\tau)}(1+\tau)^{-((n / 4)-(k / 2))} d \tau \\
\leq & C \mathscr{W}^{2}(t)(1+t)^{-((k+1) / 2)} \eta(t) .
\end{aligned}
$$

Collecting (46)-(50), which yields

$$
\begin{aligned}
\left\|\partial_{x}^{k} u_{t}(t)\right\|_{L^{2}} \leq & C E_{0}(1+t)^{-((k+1) / 2)} \\
& +C \mathscr{W}^{2}(t)(1+t)^{-((k+1) / 2)} \eta(t) .
\end{aligned}
$$

Substituting the estimate $\mathscr{W}(t)(t) \leq C E_{0}$ into (51), we arrive at the desired estimate (7) for $0 \leq k \leq s$. This completes the proof of Theorem 1.

\section{Acknowledgments}

This work was supported in part by the NNSF of China (Grant no. 11101144) and Innovation Scientists and the Technicians Troop Construction Projects of Henan Province. Funding scheme for young teachers of Universities of Henan Province.

\section{References}

[1] Y. Zhang, Q. Lin, and S. Lai, "Long-time asymptotic for the damped Boussinesq equation in a circle," Journal of Partial Differential Equations, vol. 18, no. 2, pp. 97-113, 2005.

[2] S. Lai, Y. Wang, Y. Wu, and Q. Lin, "An initial-boundary value problem for a generalized Boussinesq water system in a ball," International Journal of Applied Mathematical Sciences, vol. 3, no. 2, pp. 117-133, 2006.

[3] Y. Wang, "Existence and asymptotic behavior of solutions to the generalized damped Boussinesq equation," Electronic Journal of Differential Equations, vol. 2012, pp. 1-11, 2012.

[4] Y.-X. Wang and Z. Wei, "Global existence and asymptotic behavior of solutions to Cahn-Hilliard equation with inertial term," International Journal of Mathematics, vol. 23, no. 9, Article ID 1250087, 14 pages, 2012.

[5] S. Wang and H. Xu, "On the asymptotic behavior of solution for the generalized IBq equation with hydrodynamical damped term," Journal of Differential Equations, vol. 252, no. 7, pp. 42434258, 2012.

[6] G.-w. Chen and C.-s. Hou, "Initial value problem for a class of fourth-order nonlinear wave equations," Applied Mathematics and Mechanics, vol. 30, no. 3, pp. 391-401, 2009.

[7] Y.-Z. Wang, "Global existence and asymptotic behaviour of solutions for the generalized Boussinesq equation," Nonlinear Analysis. Theory, Methods \& Applications A, vol. 70, no. 1, pp. 465-482, 2009.

[8] Y. Z. Wang and X. Y. Chen, "Existence and uniqueness of global solutions to a class of Boussinesq type equations," Chinese Annals of Mathematics A, vol. 32, no. 5, pp. 565-578, 2011.

[9] Y.-Z. Wang, F. Liu, and Y. Zhang, "Global existence and asymptotic behavior of solutions for a semi-linear wave equation," Journal of Mathematical Analysis and Applications, vol. 385, no. 2, pp. 836-853, 2012.

[10] Y.-Z. Wang and Y.-X. Wang, "Global existence and asymptotic behavior of solutions to a nonlinear wave equation of fourthorder," Journal of Mathematical Physics, vol. 53, no. 1, Article ID 013512, 13 pages, 2012.

[11] T. Li and Y. Chen, Nonlinear Evolution Equations, Scientific Press, 1989, (Chinese).

[12] S. Zheng, Nonlinear Evolution Equations, vol. 133 of Monographs and Surveys in Pure and Applied Mathematics, Chapman \& Hall/CRC, Boca Raton, Fla, USA, 2004. 


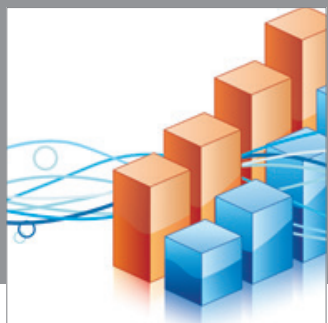

Advances in

Operations Research

mansans

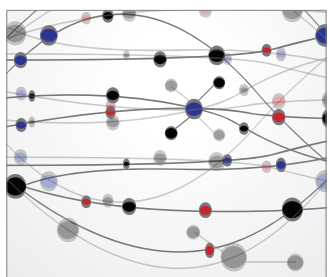

The Scientific World Journal
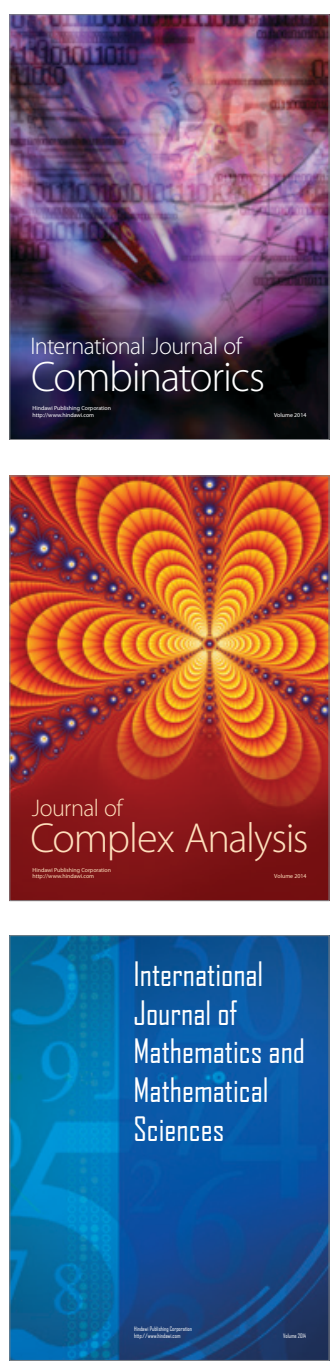
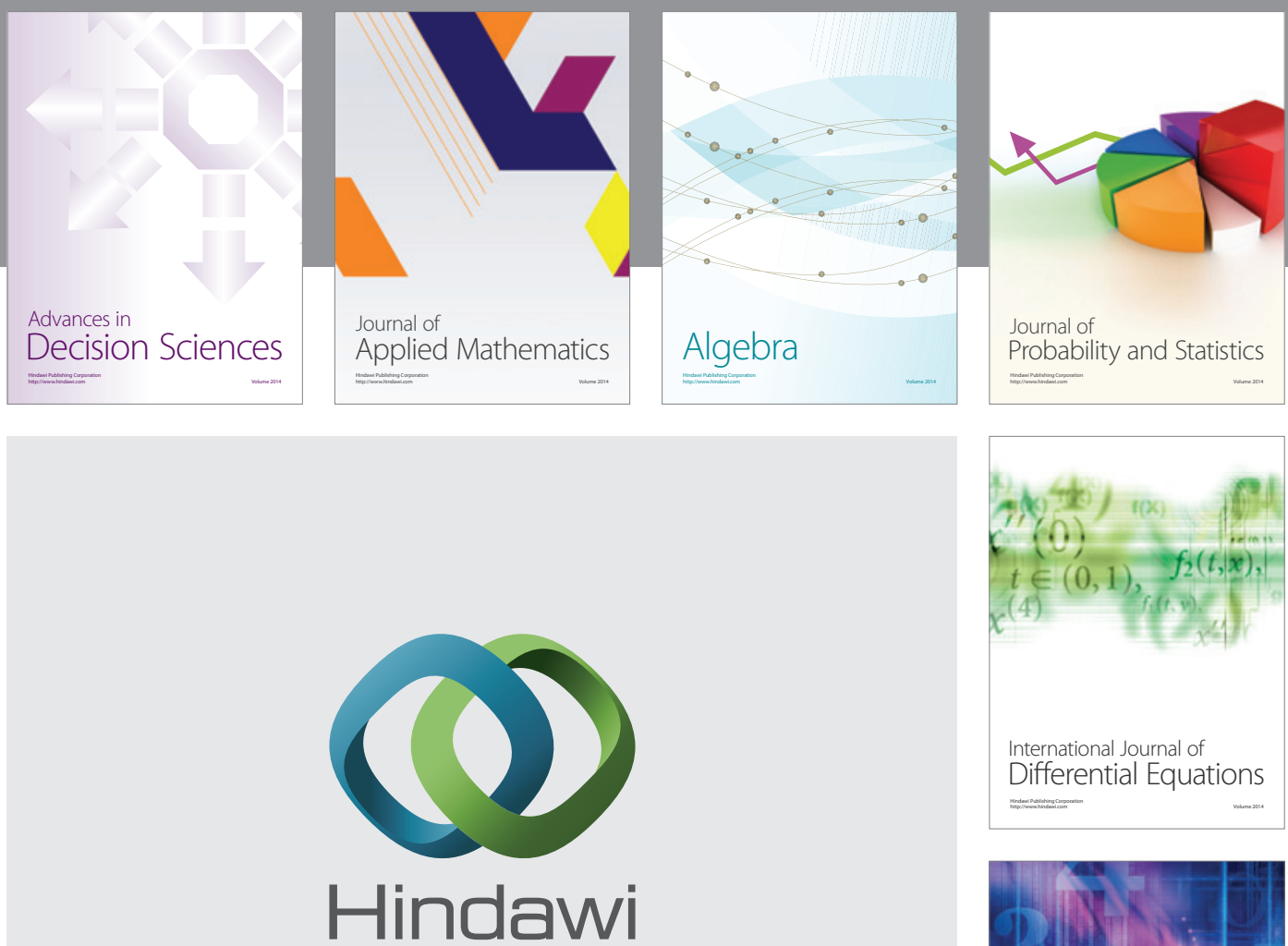

Submit your manuscripts at http://www.hindawi.com
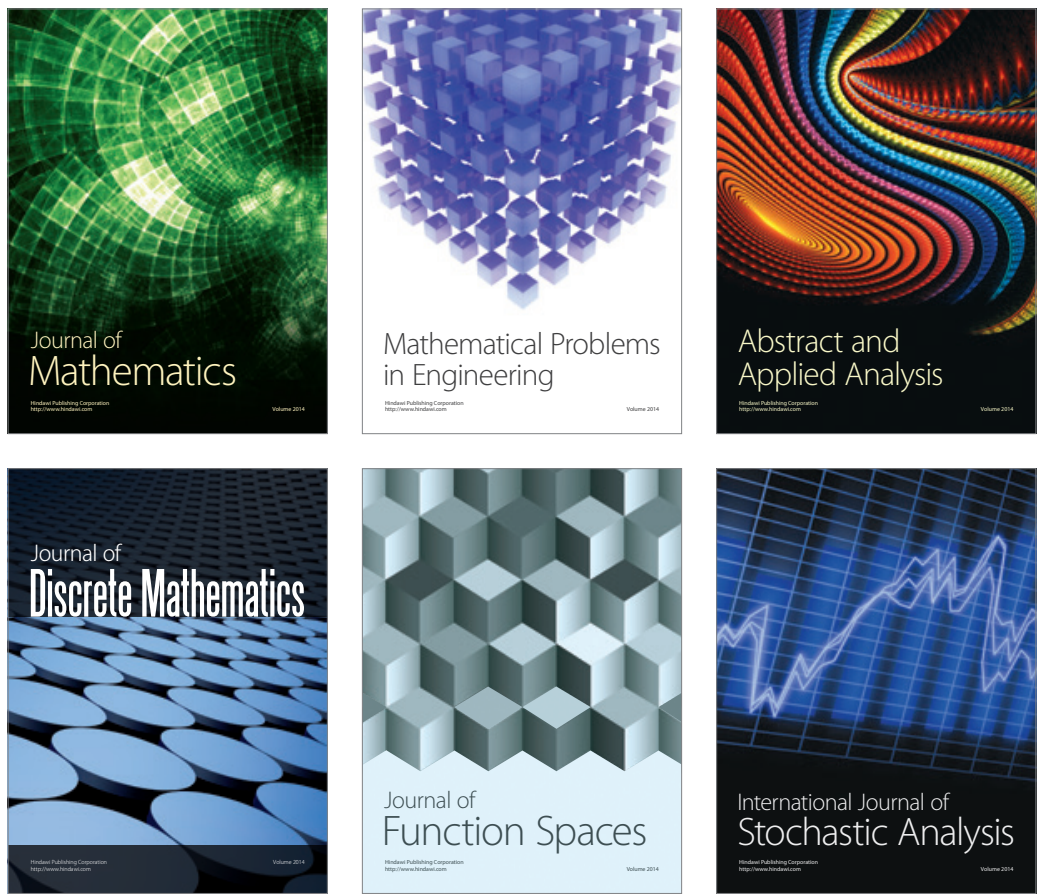

Journal of

Function Spaces

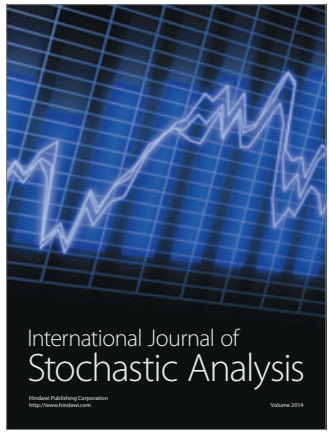

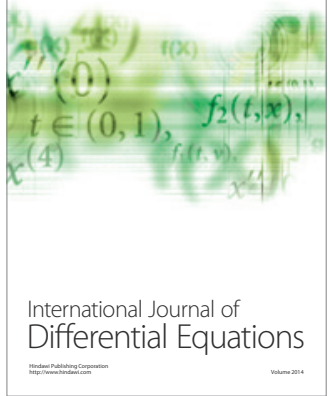
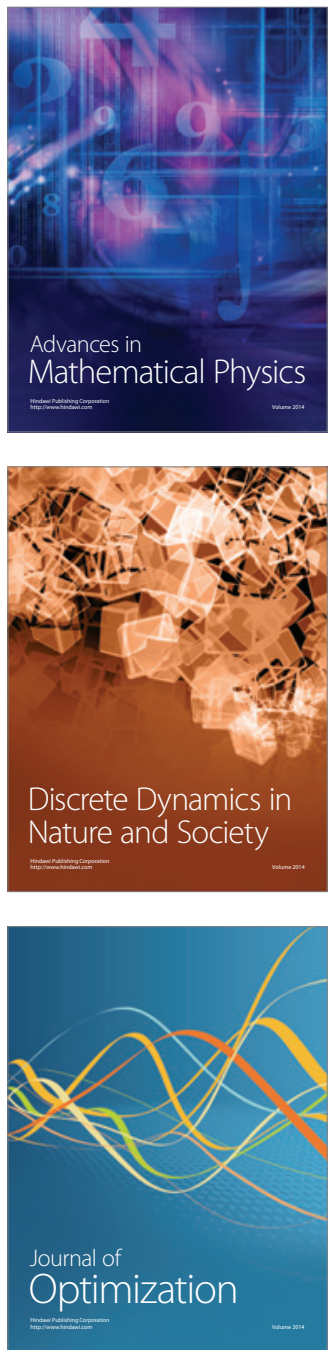